Article

\title{
Control of the Epithelial-to-Mesenchymal Transition and Cancer Metastasis by Autophagy-Dependent SNAI1 Degradation
}

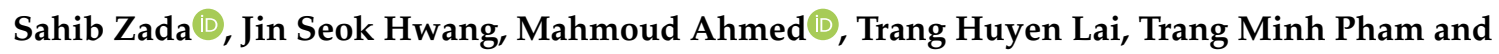 \\ Deok Ryong Kim *(D) \\ Department of Biochemistry and Convergence Medical Sciences, Institute of Health Sciences, Gyeongsang \\ National University School of Medicine, JinJu 527-27, Korea; s.zada.qau@gmail.com (S.Z.); \\ cloud8104@naver.com (J.S.H.); ma7moud_sha3ban@hotmail.com (M.A.); \\ tranghuyen20493@gmail.com (T.H.L.); phamminhtrang010895@gmail.com (T.M.P.) \\ * Correspondence: drkim@gnu.ac.kr; Tel.: +82-55-772-8054; Fax: +82-55-772-8059
}

Received: 6 December 2018; Accepted: 5 February 2019; Published: 6 February 2019

\begin{abstract}
Autophagy, an intracellular degradation process, is essential for maintaining cell homeostasis by removing damaged organelles and proteins under various conditions of stress. In cancer, autophagy has conflicting functions. It plays a key role in protecting against cancerous transformation by maintaining genomic stability against genotoxic components, leading to cancerous transformation. It can also promote cancer cell survival by supplying minimal amounts of nutrients during cancer progression. However, the molecular mechanisms underlying how autophagy regulates the epithelial-to-mesenchymal transition (EMT) and cancer metastasis are unknown. Here, we show that starvation-induced autophagy promotes Snail (SNAI1) degradation and inhibits EMT and metastasis in cancer cells. Interestingly, SNAI1 proteins were physically associated and colocalized with LC3 and SQSTM1 in cancer cells. We also found a significant decrease in the levels of EMT and metastatic proteins under starvation conditions. Furthermore, ATG7 knockdown inhibited autophagy-induced SNAI1 degradation in the cytoplasm, which was associated with a decrease in SNAI1 nuclear translocation. Moreover, cancer cell invasion and migration were significantly inhibited by starvation-induced autophagy. These findings suggest that autophagy-dependent SNAI1 degradation could specifically regulate EMT and cancer metastasis during tumorigenesis.
\end{abstract}

Keywords: autophagy; cancer metastasis; EMT; LC3; SNAI1

\section{Introduction}

Autophagy is a cellular process that sequesters protein aggregates and damaged intracellular organelles into double-membraned autophagosomes in the cytoplasm, where these materials are degraded and recycled by a lysosome-dependent process. This phenomenon is intrinsically adopted by cancer cells to support themselves in a metabolically deficient microenvironment and maintain cellular homeostasis [1-4]. Autophagy plays a context-dependent role in cancer occurrence and progression. It increases genomic stability by removing damaged organelles and proteins, which enhances genomic stability and prevents tumorigenesis. Autophagy also promotes the survival of cancer cells under metabolic stress and improves resistance to unfavorable conditions. Therefore, autophagy represents a powerful survival strategy for cancer cells exposed to intrinsic or extrinsic stressors [5-7].

Cancer metastasis is a multistep process in which cells migrate from a primary site to a distant location for colonization, and it is a major cause of cancer-related deaths [8-10]. Metastasis requires cancer cells to detach from a primary site, migrate through the bloodstream, invade stromal tissues, 
and further proliferate in secondary tissues [9-13]. EMT plays a crucial role in the initiation of cancer metastasis and is characterized by the loss of epithelial properties that leads to mesenchymal characteristics and thereby increases cell mobility and resistance to apoptosis [14-16]. However, the role of autophagy in the regulation of EMT and metastasis-related proteins during tumorigenesis is unclear, although many studies suggest possible connections to cancer progression.

SNAI1/SNAIL is a key transcription factor that controls initiation of EMT [17-19]. It inhibits the activity of the E-cadherin/CDH1 gene and subsequently promotes metastasis of most cancers [20-23]. Increased levels of SNAI1 also induce the self-renewal program of cancer stem-like cells by upregulating stemness factors that cause drug resistance [24-26]. In addition, SNAI1 has been shown to inhibit the activity of p53, which plays a crucial role in tumor suppression $[19,24,27]$. These findings suggest that the inactivation of SNAI1 proteins could be a potential target for the development of cancer therapies.

MAP1LC3/LC3 is a key protein involved in autophagosome formation; it regulates autophagy through its direct interaction with SQSTM1/p62. The sequence of LC3 is evolutionarily conserved from yeast to mammals. Mutations in LC3 that abrogate its ability to bind SQSTM1 cause cytotoxicity due to the excessive accumulation of SQSTM1 [28-30]. LC3-SQSTM1 interactions are required for degradation of polyubiquitylated protein aggregates by autophagy [30]. However, autophagy-mediated degradation of some long-lived proteins is unaffected by knockdown of the SQSTM1 gene [31], indicating that autophagy can degrade proteins, not only via LC3-SQSTM1 interactions but also through direct interactions with LC3. Indeed, previous studies have suggested that autophagy-dependent protein degradation might be associated with cancer progression [32,33]. However, the mechanistic basis underlying how autophagy regulates EMT and metastasis is not clear.

In this study, we show that starvation-induced autophagy causes the specific degradation of SNAI1 via LC3-SQSTM1 interactions. In addition, autophagy inhibits the translocation of SNAI1 to the nucleus as well as the migration and invasion of cancer cells, suggesting that degradation of SNAI1 by autophagy is a critical process that controls tumorigenesis. Furthermore, we suggest that targeting autophagy-dependent SNAI1 degradation is a promising strategy for the development of cancer therapies.

\section{Materials and Methods}

\subsection{Reagents}

Dulbecco's modified Eagle's medium (DMEM, 11995-065), Roswell Park Memorial Institute 1640 Medium (RPMI-1640 (11875-119), Hank's buffered salt solution (HBSS, 14025-092), and fetal bovine serum (FBS; 16000-044) were purchased from Gibco and Life Technologies. Chloroquine (C6628) was purchased from Sigma-Aldrich (St. Louis, MO, USA). Rapamycin (R-5000) and bafilomycin A1 were purchased from LC Laboratories (Woburn, MA, USA). Primary antibodies against LC3A/B (12741), SNAI1 (3879), TCF8/Zeb1 (3396), N-cadherin (13116), SQSTM1 (5114), phospho-ULK1 (Ser555; 5869), phospho-ULK1 (Ser757; 14202), AMPK $\alpha$ (2532), AMPK $\alpha$ T172 (2531), mTOR (2983), and phospho-mTOR (Ser2448; 2971) were from Cell Signaling Technology (Beverly, MA, USA). MAP1LC3 (SC-376404), SQSTM1/p62 (SC-28359), vimentin (sc-6601), E-cadherin (SC-7870), $\alpha$-tubulin (SC-5286), and APG7 (SC-376212) were from Santa Cruz Biotechnology (Santa Cruz, CA, USA). SNAI1 (ab 53519) was from Abcam (Cambridge, MA, USA), and $\beta$-actin (A5441) was from Sigma-Aldrich. Secondary antibodies against rabbit IgG (STAR208P) and mouse IgG (STAR117P) were purchased from Bio-Rad (Hercules, CA, USA). Secondary antibodies for immunocytochemistry (FITC and TRITC) were from Santa Cruz. Protein A/G PLUS agarose immunoprecipitation reagent (sc2003) was from Santa Cruz Biotechnology. Matrigel (Corning \# 344235), propidium iodide (PI), and ProLong ${ }^{\mathrm{TM}}$ Diamond antifade mountant with DAPI (\# p36966) were from Invitrogen (Carlsbad, CA, USA). G488 was purchased from Thermo Scientific (Rockford, IL, USA). 


\subsection{Cell Culture}

HeLa cells were cultured in DMEM containing 10\% FBS. H1299 cells were cultured in the RPMI- 1640 medium containing $10 \%$ FBS. All cells were grown at $37{ }^{\circ} \mathrm{C}$ in a humidified atmosphere incubator of $95 \%$ air and $5 \% \mathrm{CO}_{2}$.

\subsection{Western Blot Analysis}

Total proteins were extracted with a cell lysis buffer supplemented with a protease and phosphatase inhibitor cocktail (Halt ${ }^{\mathrm{TM}}$ Protease and Phosphatase Inhibitor Cocktail $100 \times$, Thermo Scientific). Protein concentrations were determined using the Pierce BCA Protein Assay Kit (Thermo Scientific). Total protein lysates (30 $\mu \mathrm{g}$ ) were separated by $10 \%$ SDS-PAGE, and the target proteins were specifically detected by western blotting using the indicated antibodies. Proteins were visualized with the enhanced chemiluminescence detection reagent (Thermo Scientific). All data were normalized to $\beta$-actin levels.

\subsection{Coimmunoprecipitation Assay}

Coimmunoprecipitation was performed as described in the materials (protein G agarose, sc11243233001). Briefly, HeLa cells were treated with HBSS for $4 \mathrm{~h}$. Then, cells were harvested and lysed in Pierce IP lysis buffer (Thermo Scientific) supplemented with a protease and phosphatase inhibitor cocktail. Cell lysates were incubated with anti-LC3 or -SQSTM1 antibodies overnight at $4{ }^{\circ} \mathrm{C}$ with gentle rotation. Then, $50 \mu \mathrm{L}$ of protein $\mathrm{G}$ agarose was added to the antibody mixture and incubated at $4{ }^{\circ} \mathrm{C}$ for $4 \mathrm{~h}$ with gentle shaking. Immunoprecipitates were washed with lysis buffer three times. The bound proteins were eluted by boiling the beads in $2 \times$ SDS gel loading buffer and subjected to SDS-PAGE. Specific proteins were detected by western blotting using the indicated primary antibodies.

\subsection{Plasmid Transfection}

HeLa and H1299 cells were transfected with ATG7 shRNA or control plasmids using Lipofectamine 3000 (Invitrogen), as described in the manufacturer's protocol. After incubation for $24 \mathrm{~h}$ in fresh media, cells were starved in HBSS for $4 \mathrm{~h}$, and total proteins were extracted and analyzed by western blotting as described above.

\subsection{Immunofluorescence Staining}

Cells were cultured on coverslips for $24 \mathrm{~h}$ and then starved in HBSS for $4 \mathrm{~h}$. Cells were fixed with $4 \%(w / v)$ paraformaldehyde for 30 min and permeabilized with PBS containing $0.1 \%$ Triton X-100 for $20 \mathrm{~min}$ at room temperature. Cells were blocked with $5 \%$ horse serum in PBS for $1 \mathrm{~h}$ and then incubated with primary antibodies overnight at $4{ }^{\circ} \mathrm{C}$. After washing with PBS, cells were incubated with FITC- or TRITC-conjugated secondary antibodies (1:50 in PBS) at room temperature for $90 \mathrm{~min}$. The slides were washed twice with PBS for $5 \mathrm{~min}$. Glass coverslips were mounted onto glass slides using mounting medium containing DAPI. The images were captured by confocal microscopy (FV-1000; Olympus, Tokyo).

\subsection{Subcellular Fractionation}

Cells were cultured to 70-80 \% confluence and then starved in HBSS for $4 \mathrm{~h}$. Cells were washed with ice-cold PBS and then scraped. After centrifugation, cells were resuspended in RSB (10 mM Tris, $\mathrm{pH} 7.4,10 \mathrm{mM} \mathrm{NaCl}$, and $6 \mathrm{mM} \mathrm{MgCl}_{2}$ ) and incubated for $10 \mathrm{~min}$ on ice. Cells were then incubated in RSB containing $50 \mu \mathrm{M} \mathrm{DDT}, 10 \mathrm{mM} \mathrm{NaF}$, and $1 \mathrm{mM} \mathrm{NaVO}_{4}$ on ice for $15 \mathrm{~min}$. After centrifugation, the supernatants and pellets were collected as the cytosolic and nuclear fractions, respectively. Pellets were resuspended in three volumes of buffer C (20\% glycerol, $20 \mathrm{mM}$ HEPES, pH 7.9, $420 \mathrm{mM} \mathrm{NaCl}, 1.5 \mathrm{mM}$ 
$\mathrm{MgCl}_{2}, 10 \mathrm{mM} \mathrm{NaF}$, and $1 \mathrm{mM} \mathrm{NaVO}_{4}$ ) and incubated on ice for $30 \mathrm{~min}$. Supernatants were collected as nuclear proteins after high-speed centrifugation.

\subsection{Wound-Healing (Scratch) Assay}

Cells were grown to confluence in a 60-mm dish. Cells were treated either with $100 \mathrm{nM}$ rapamycin and HBSS to induce autophagy or with $10 \mathrm{nM}$ bafilomycin A1 to inhibit autophagy. A "wound" line was scratched into the cell monolayer with a sterile $1000-\mu \mathrm{L}$ pipette tip in three separate places. The widths of the wound areas were photographed and measured under an inverted, phase-contrast microscope (Nikon, $50 \times$ magnification) to assess cell migration at $0,24,48$, and $72 \mathrm{~h}$ after scratching. Distances covered by the cells from the initial wound point were measured with a ruler in centimeters.

\subsection{Cell Invasion Assay}

A transwell insert (Corning) with an 8 - $\mu$ m pore size was used for the two-chamber migration assay. The upper surface of the transwell insert was coated with Matrigel (BD Biosciences(San jose, CA, USA); $50 \mathrm{mg}$ / filter), and HeLa cells treated with $100 \mathrm{nM}$ rapamycin, HBSS, or $10 \mathrm{nM}$ bafilomycin A1 were added. Cells were seeded in serum-free medium in the upper chamber. Serum-containing medium $(10 \% \mathrm{FBS})$ was used in the lower chamber as a chemoattractant. After $36 \mathrm{~h}$, cells that had invaded the bottom chamber were fixed with 3.7\% formaldehyde, permeabilized with 100\% methanol, stained with PI, and photographed under a microscope. Cells were quantified by counting the number of cells in 10 randomly selected areas.

\subsection{Statistical Analysis}

Each experiment was conducted independently at least three times, and results were expressed as the mean \pm standard deviation (S.D.). The differences between two groups were assessed using a two-tailed Student's t-test. One-way analysis of variance statistics was used to compare the means of three groups or more, followed by a Tukey's multiple comparison test. Values of $p$ less than 0.05 and $p$ less than 0.01 were considered significant.

\section{Results}

\subsection{Autophagy Specifically Regulates SNAI1 Degradation in Cancer Cells}

SNAI1 is a key regulator of EMT and cancer metastasis. Thus, the intracellular levels of SNAI1 should be specifically regulated during cancer progression. Indeed, proteasomes degrade ubiquitinated SNAI1 in cancer cells [32-36]. To elucidate the mechanism underlying how autophagy contributes to SNAI1 degradation in cancer cells, we examined the protein levels of SNAI1 in cancer cells after autophagy was induced by starvation or treatment with rapamycin $(100 \mathrm{nM})$. In both H1299 (lung cancer, Figure 1A,B) and HeLa (cervical cancer, Figure 1C,D) cell lines, SNAI1 protein levels were significantly decreased when autophagy was induced by rapamycin or starvation in HBBS. This autophagy-dependent SNAI1 degradation was significantly decreased by the addition of $20 \mu \mathrm{M}$ chloroquine, an autophagy inhibitor. We further verified the induction of autophagy using autophagy markers, such as LC3-II formation, SQSTM1 degradation, ULK1 activity (ULK1 is activated by phosphorylation at S555 and inhibited by phosphorylation at S757), AMPK activation by phosphorylation at T172, and mTOR activation by phosphorylation at S2448. These marker proteins were appropriately activated or repressed in an autophagy-dependent manner (Figure 1A,C). 
A

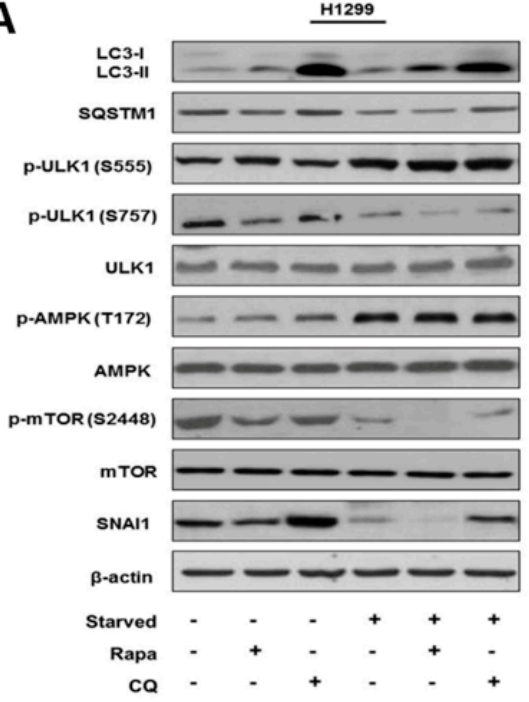

B

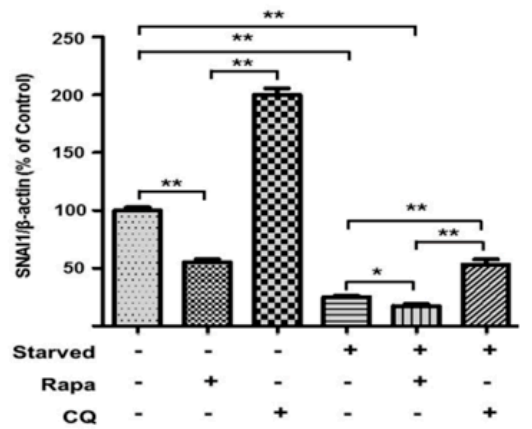

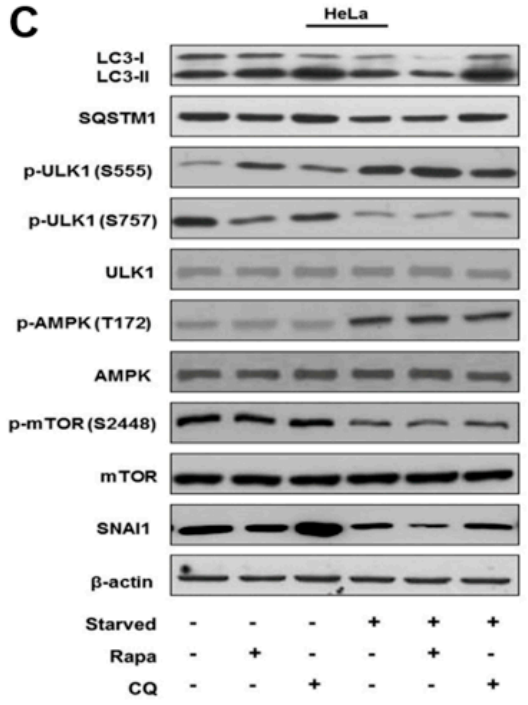

D

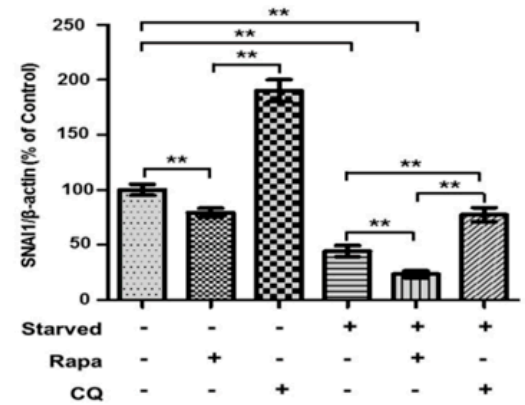

Figure 1. Autophagy stimulates SNAI1 degradation in cancer cells. H1299 (A) and HeLa (C) cells were treated with $100 \mathrm{nM}$ rapamycin (Rapa) or starved in HBSS medium for $4 \mathrm{~h}$ to induce autophagy in the presence or absence of $20 \mu \mathrm{M}$ chloroquine (CQ). After lysis, total cell extracts (30 $\mu \mathrm{g})$ were separated by $10 \%$ or $12 \%$ SDS-PAGE and analyzed by western blotting using primary antibodies against LC3, SQSTM1, p-ULK1-S555, p-ULK1-S757, p-AMPK-T172, AMPK, p-mTOR-S2248, mTOR, and SNAI1. $\beta$-actin was used as a loading control. The levels of SNAI1 in H1299 (B) and HeLa cells (D) were quantified using NIH ImageJ software. Data represent the mean $( \pm$ S.D. $)$ of three independent experiments $\left({ }^{*} p<0.05\right.$ and $\left.{ }^{* *} p<0.01\right)$.

\subsection{Autophagy-Dependent SNAI1 Degradation Is Required for LC3-SQSTM1 Interactions}

Autophagy non-selectively eliminates bulky proteins and organelles, and in some instances, this process can be selective, such as the interaction that occurs between LC 3 and partner proteins that have specific amino acid sequences containing an LC3-interacting region (LIR) motif [31,37]. SQSTM1, a well-known LC3-interacting adaptor protein, is involved in the degradation of associated proteins through this selective autophagy. In the context of selective SNAI1 degradation by autophagy, we used immunoprecipitation to examine the physical interaction between SNAI1 and LC3 and/or SQSTM1 under starvation conditions. Indeed, these three proteins were coimmunoprecipitated by anti-LC3 or anti-SQSTM1 antibodies, and their interaction increased in starved cells (Figure 2A,B). The SNAI1 interaction with LC3 or SQSTM1 was also evaluated by colocalization using immunocytochemistry (Figure 2C,E) and assessed quantitatively (Figure 2D,F). SNAI1 similarly colocalized with both LC3 and SQSTM1. These results indicate that autophagy degrades SNAI1 via an LC3 and/or SQSTM1-dependent manner. 
A

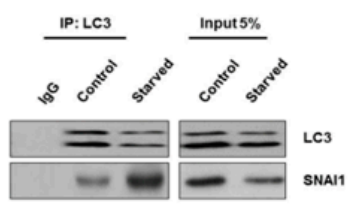

C

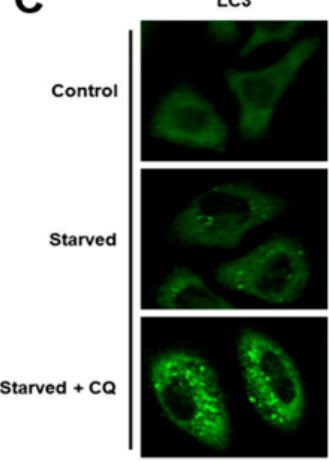

E

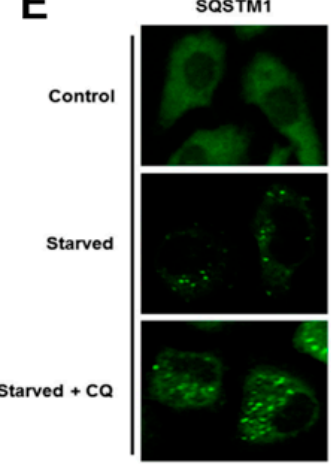

SNAI1

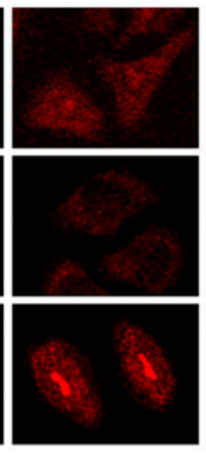

SNA11

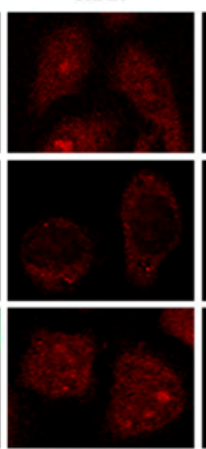

B

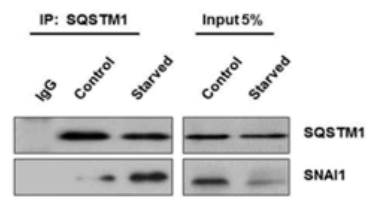

DAPI

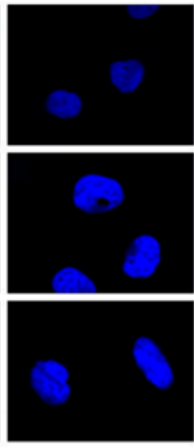

DAPI

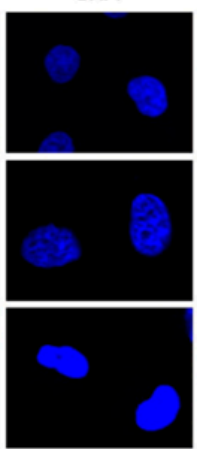

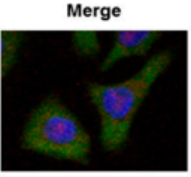

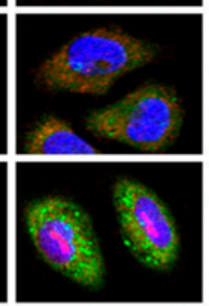

Merge

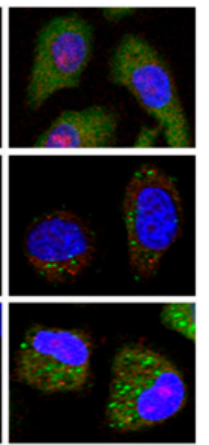

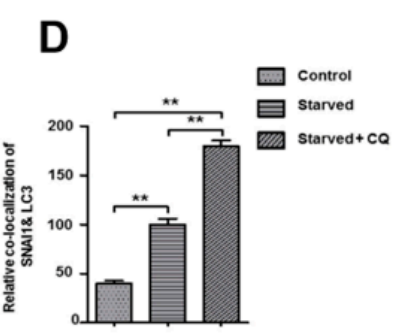

$\mathbf{F}$

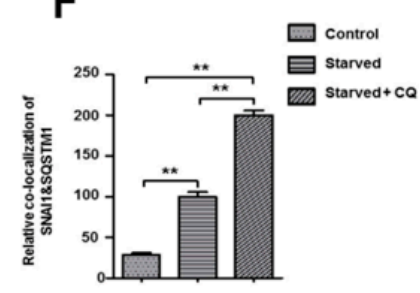

Figure 2. Possible interactions between SNAI1 and LC3 or SQSTM1/p62. (A,B) Coimmunoprecipitation. Control or starved HeLa cell lysates were immunoprecipitated by anti-LC3 (A) or anti-SQSTM1 (B) antibodies in $30 \mu \mathrm{L}$ protein $\mathrm{G}$ agarose beads. After washing, the bound proteins were analyzed by western blotting using the indicated antibodies. Whole-cell extracts ( $5 \%$ input) were also assessed by western blotting. IgG indicates nonspecific mouse antibody as a negative control. (C,E) Immunocytochemistry. HeLa cells were cultured on coverslips for $24 \mathrm{~h}$ and then starved with HBSS for $4 \mathrm{~h}$. After fixing cells with paraformaldehyde, cells were incubated for $24 \mathrm{~h}$ at $4{ }^{\circ} \mathrm{C}$ with rabbit polyclonal anti-SNAI1 plus mouse monoclonal anti-LC3 (C) or mouse monoclonal anti-SQSTM1 (E) antibodies. After washing, secondary antibodies (FITC-conjugated anti-mouse antibody or TRITC-conjugated anti-rabbit antibody) were applied to cells. The glass slides were mounted using a mounting medium containing DAPI (to stain nuclei), and all images were captured by confocal microscopy (Olympus FV-1000). (D,F) Colocalization of SNAI1 with LC3 (D) and SQSTM1 (F) was quantified using NIH ImageJ software. Data represent the mean ( \pm S.D.) of three independent experiments $\left.{ }^{* *} p<0.01\right)$.

\subsection{Autophagy-Dependent SNAI1 Degradation Is Associated with EMT and Cancer Metastasis}

During EMT and cancer metastasis, the levels of proteins such as N-cadherin, Zeb1, and vimentin significantly increase while the amount of E-cadherin decreases due to SNAI1-dependent transcriptional regulation [17-23]. To investigate how autophagy-dependent SNAI1 degradation affects the expression of EMT and metastasis-related proteins, we induced autophagy in two different cancer cell lines (H1299 and HeLa) with HBSS for $4 \mathrm{~h}$ in the presence or absence of $20 \mu \mathrm{M}$ chloroquine. In both cell types, autophagy was similarly activated by HBSS treatment. Activation of ULK1 and AMPK and degradation of SQSTM1 were observed after starvation. Moreover, the levels of SNAI1 proteins were dependent on the induction of autophagy (Figure 3A-C), and there was a large decrease in the levels of mesenchyme-related proteins (including N-cadherin, and Zeb1) upon 
starvation-induced autophagy (Figure 3B,C). However, vimentin was not significantly changed in H1299 cells (Figure 3B,C). The expression levels of these proteins significantly increased by inhibiting autophagy with $20 \mu \mathrm{M}$ chloroquine. In contrast, the levels of epithelial proteins, such as E-cadherin, were highly increased by the induction of autophagy under starvation and decreased after treatment with $20 \mu \mathrm{M}$ chloroquine in all cancer cell lines (Figure 3). We further examined the expression levels of SNAI1 using immunocytochemistry. Indeed, the intensity of SNAI1 was decreased in starved cells but increased after treatment with chloroquine (Figure 3D,E), suggesting that the intracellular levels of SNAI1 are specifically regulated by autophagy and functionally associated with the regulation of cancer progression.

A

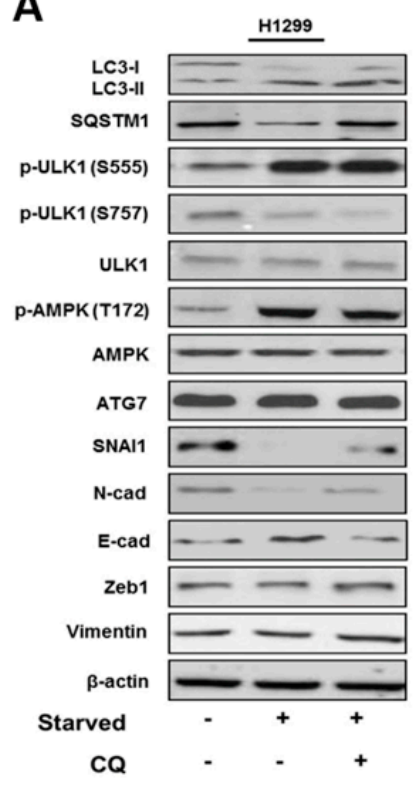

D

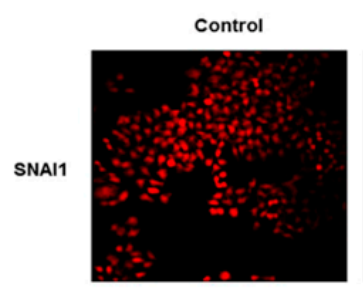

HeLa

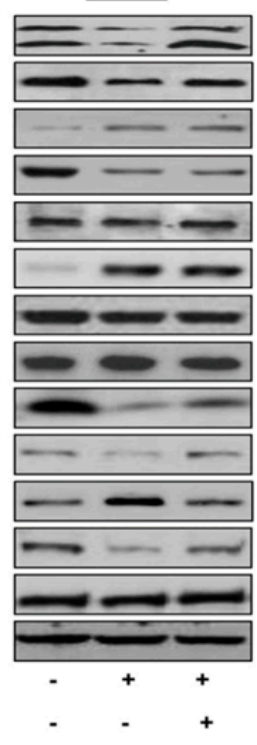

Starved

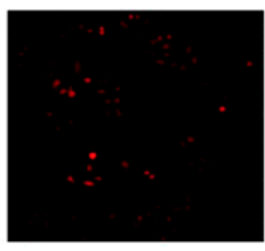

B

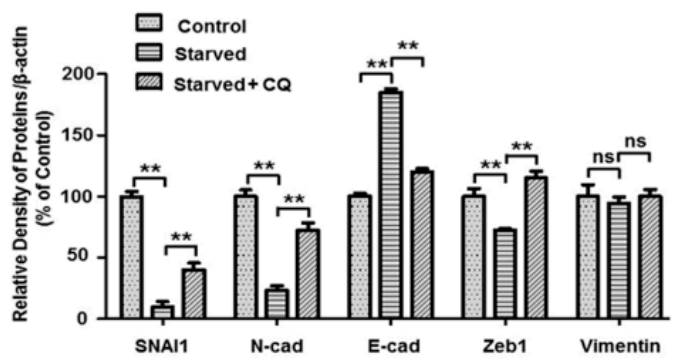

C

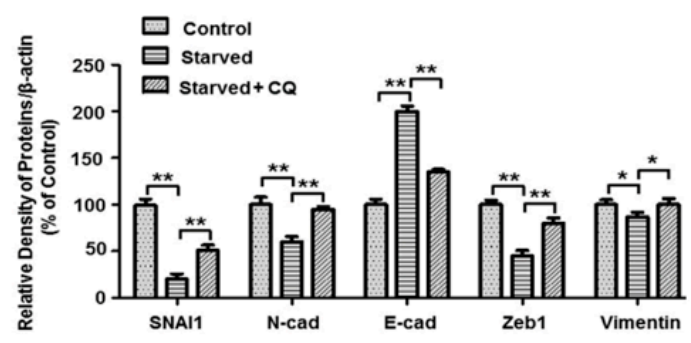

E

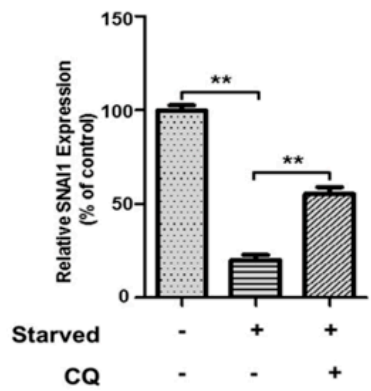

Figure 3. Autophagy-induced SNAI1 degradation is associated with the expression of EMT- and cancer metastasis-related proteins. (A) H1299 (left) and HeLa (right) cells were starved with HBSS for $4 \mathrm{~h}$ in the presence or absence of $20 \mu \mathrm{M}$ chloroquine (CQ). After lysis, total-cell extracts $(30 \mu \mathrm{g})$ were separated by $10 \%$ or $12 \%$ SDS-PAGE and analyzed by western blotting using primary antibodies against LC3, SQSTM1, p-ULK1-S555, p-ULK1-S757, p-AMPK-T172, AMPK, p-mTOR-S2248, mTOR, SNAI1, N-cadherin, E-cadherin, Zeb1, and vimentin. $\beta$-actin was used as a loading control. (B,C) The levels of each EMT-related protein (SNAI1, N-cadherin, Zeb1, and vimentin) in H1299 (B) or HeLa (C) cells were quantified using NIH ImageJ software. $\beta$-actin was used as a loading control. Data represent the mean ( \pm S.D.) of three independent experiments ${ }^{*} p<0.05$ and $\left.{ }^{* *} p<0.01\right)$. (C,D) Immunocytochemistry of SNAI1. HeLa cells were treated with HBSS with or without $20 \mu \mathrm{M} \mathrm{CQ}$ for $4 \mathrm{~h}$. The expression levels of SNAI1 (red) in cells were determined by immunocytochemistry (C). Images were captured from at least 20 different areas by florescence microscopy (Olympus BX51-DSU), and the immunofluorescence of SNAI1 was quantified using NIH ImageJ software (D). Data represent the mean $( \pm$ S.D.) of three independent experiments $(* * p<0.01)$. 


\subsection{ATG7 Knockdown Rescues Starvation-Induced SNAI1 Degradation}

Because ATG7 plays a crucial role in the autophagy process, knockdown of the ATG7 gene was shown to cause a severe defect in autophagy via LC3 lipidation with phosphatidylethanolamine [38]. To investigate how the knockdown of the ATG7 gene affects SNAI1 degradation, we transiently transfected plasmids containing ATG7 shRNA and control shRNA into two cancer cell lines (HeLa and H1299) and incubated the cells for $24 \mathrm{~h}$. The expression levels of ATG7 in both HeLa and H1299 cells were significantly decreased after treatment with ATG7 shRNA, and starvation-induced autophagy was subsequently found to be defective after ATG7 knockdown (Figure 4). Furthermore, autophagy defects induced by the ATG7 knockdown significantly inhibited the degradation of SNAI1 in both cancer cell lines (Figure 4), indicating that ATG7-dependent autophagy is crucial for controlling the protein levels of SNAI1 in cancer cells.

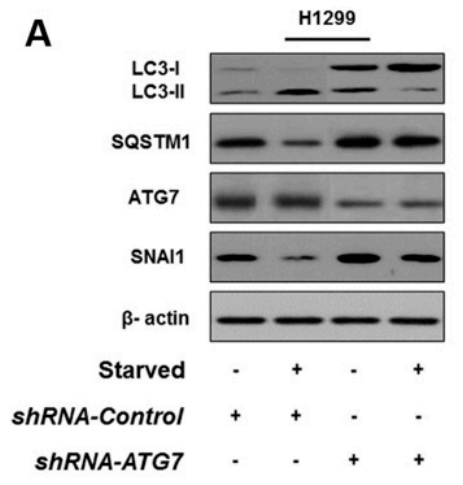

B

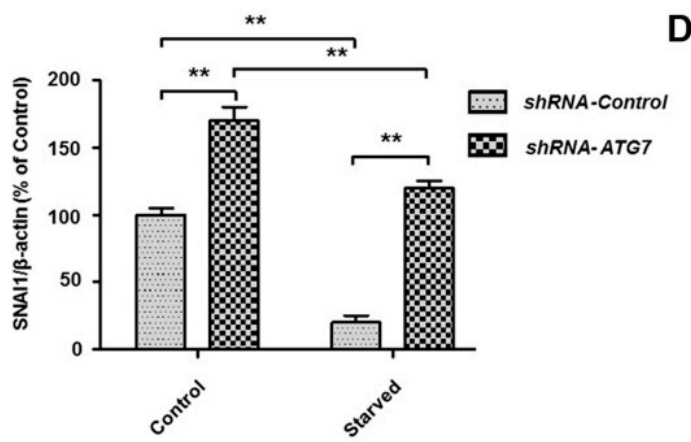

C

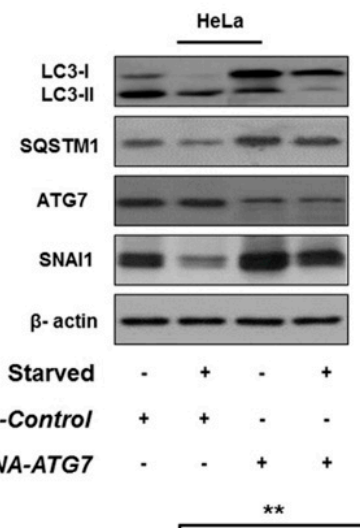

D

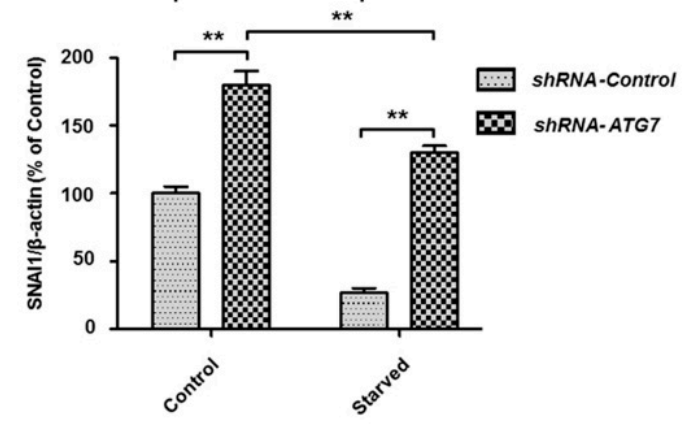

Figure 4. ATG7 knockdown rescues SNAI1 degradation. H1299 (A) or HeLa (C) cells were transiently transfected with ATG7 shRNA and control shRNA plasmids using Lipofectamine 3000 and incubated for $24 \mathrm{~h}$. Cells were then starved with HBSS for $4 \mathrm{~h}$. Total-cell proteins $(30 \mu \mathrm{g})$ were separated by $10 \%$ or $12 \%$ SDS-PAGE and analyzed by western blotting using antibodies against LC3, SQSTM1, ATG7, and SNAI1. $\beta$-actin was used as a loading control. (B,D) Quantification of SNAI1. The levels of SNAI1 in H1299 cells (B) and HeLa cells (D) were quantified using NIH ImageJ software. $\beta$-actin was used as a loading control. Data represent the mean $\left( \pm\right.$ S.D.) of three independent experiments $\left({ }^{* *} p<0.01\right)$.

\subsection{Starvation-Induced Autophagy Inhibits SNAI1 Translocation to the Nucleus}

SNAI1, a transcription factor, is translocated into the nucleus where it inhibits the transcription of epithelial markers such as E-cadherin/CDH1 and promotes metastasis of most cancers [20-23]. In this study, we examined the intracellular levels of SNAI1 in both the cytosol and nucleus when HeLa cells were starved in HBSS in the presence or absence of $20 \mu \mathrm{M}$ chloroquine. In both the cytosol and nucleus, the levels of SNAI1 were significantly decreased after starvation, although nuclear SNAI1 levels were less affected than cytosolic SNAI1 levels (Figure 5A). Similarly, SNAI1 levels in both the cytosol and nucleus recovered after the inhibition of autophagy by chloroquine (Figure 5B,C). Furthermore, according to immunocytochemical data, the nuclear localization of SNAI1 was reduced 
after cells were starved and substantially increased after treatment with chloroquine (Figure 5D,E). These data demonstrate that autophagy degrades SNAI1 in the cytoplasm and consequently inhibits SNAI1 translocation to the nucleus.

A

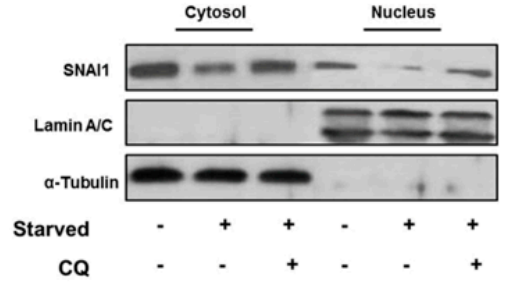

D

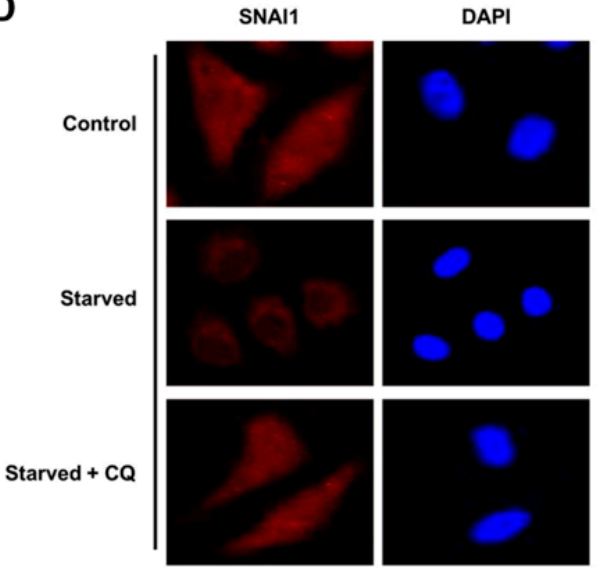

B

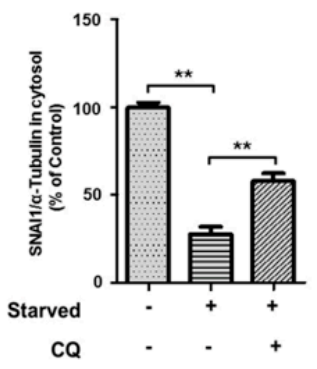

C

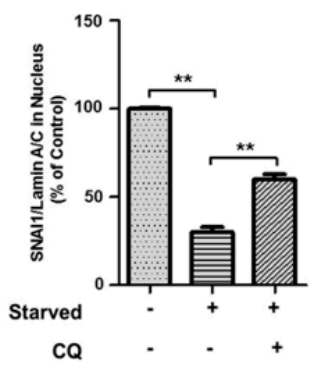

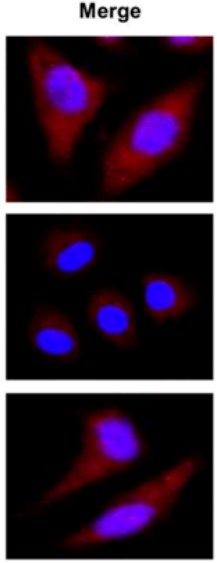

$E$

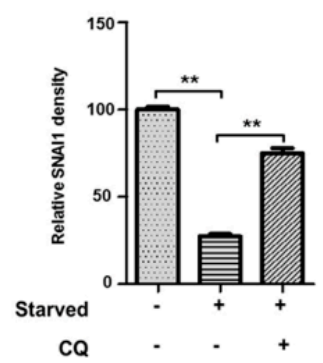

Figure 5. Autophagy-dependent degradation of SNAI1 in the cytosol inhibits SNAI1 translocation into the nucleus. (A) HeLa cells were starved in HBSS for $4 \mathrm{~h}$ with or without $20 \mu \mathrm{M}$ chloroquine (CQ). Whole cell extracts were fractionated into cytosolic and nuclear extracts as described in the methods. Each fraction was analyzed by western blotting analysis. Both $\alpha$-tubulin and lamin A/C were used as loading controls for the cytosolic and nuclear fractions, respectively. (B,C) Quantification of SNAI1. The levels of SNAI1 in the cytosol (B) and nucleus (C) were quantified using NIH ImageJ software. Data represent the mean ( \pm S.D.) of three independent experiments $\left({ }^{* *} p<0.01\right)$. (D,E) Localization of SNAI1 in the cytosol and nucleus. HeLa cells were starved in HBSS in the presence or absence of $20 \mu \mathrm{M}$ CQ. SNAI1 staining (C) in the cytosol or nucleus was determined by immunohistochemistry. Images were captured from at least 20 different areas by confocal microscopy (Olympus FV-1000), and the immunofluorescence of nuclear SNAI1 was quantified using NIH ImageJ software (D). Data represent the mean ( \pm S.D.) of three independent experiments $(* * p<0.01)$.

\subsection{Autophagy Inhibits Cancer Cell Migration and Invasion}

As described above, the intracellular levels of SNAI1 are regulated by autophagy. To investigate whether autophagy-induced SNAI1 degradation influences the migration and invasion of cancer cells, we performed scratch assays under different conditions. Autophagy was induced by treatment with $100 \mathrm{nM}$ rapamycin or HBSS (starvation), resulting in reduced wound closure activity by HeLa cancer cells. In contrast, the inhibition of autophagy with $10 \mathrm{nM}$ bafilomycin A1 largely increased the wound closure activity of HeLa cells in normal media and under starvation conditions (Figure 6A,B). In these experiments, we used $10 \mathrm{nM}$ bafilomycin A1 rather than $20 \mu \mathrm{M}$ chloroquine to inhibit autophagy because chloroquine revealed high cytotoxicity in case of long-term incubation. We also examined cell invasion under the same conditions using the Matrigel chamber assay. Induction of autophagy decreased cell invasion while its inhibition with bafilomycin A1 significantly increased the invasion activity of cancer cells (Figure 6C,D). Additionally, similar results were observed in H1299 cells (data 
not shown). These results indicate that autophagy plays an important role in cancer migration and invasion via the possible modulation of intracellular SNAI1 levels.

A

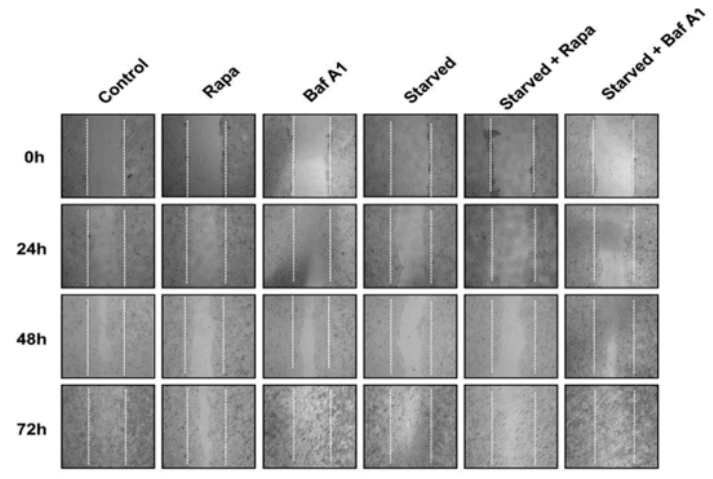

C

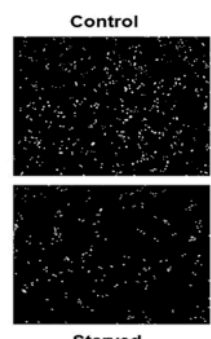

Starved

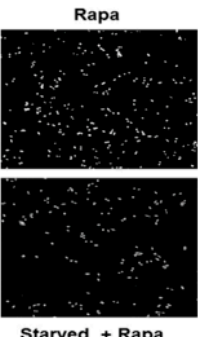

Starved + Rapa

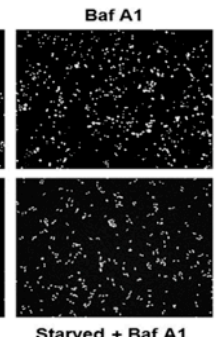

B

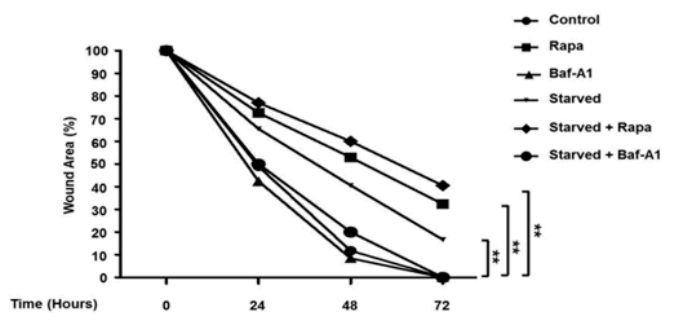

D

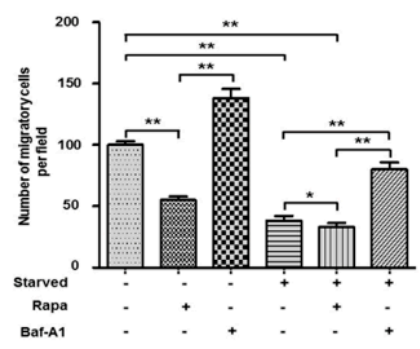

Figure 6. Autophagy inhibits migration of cancer cells. (A,B) HeLa cells were treated with either $100 \mathrm{nM}$ rapamycin (Rapa) or HBSS to induce autophagy in the presence or absence of $10 \mathrm{nM}$ bafilomycin A1 (BafA1). The migratory behavior of cancer cells under different conditions was determined by the wound-healing assay, as described in the methods, and representative images (50× magnification) indicate cell migration at the times indicated. The wound edges at $0 \mathrm{~h}$ are indicated by white dotted lines. Cell migration (B) is represented as wound closure (percentage of the average migratory distance of cells relative to the original wound edge). Data indicate the mean ( \pm S.D.) of at least three independent experiments $\left({ }^{*} p<0.05\right.$ and $\left.{ }^{* *} p<0.01\right)$. (C) The Matrigel invasion chamber assay using HeLa cells. Cells that invaded the transwell insert were stained with PI and photographed. (D) Cells were quantified in 10 randomly selected fields under a microscope (10× objective magnification). Data represent the mean $( \pm$ S.D.) of three independent experiments $(* * p<0.01)$.

\section{Discussion}

Autophagy is a process by which cellular metabolic homeostasis is maintained by delivering excess and unnecessary cytoplasmic materials to double-membraned autophagosomes, which ultimately fuse with lysosomes for the degradation of their contents [1-3]. Cancer cells utilize autophagy not only for their metabolic demands but also to escape stress-induced cell death [5-7]. Since autophagy plays multiple roles in tumor progression, it is one of the major targets of cancer therapy, although this depends on both cancer cell type and context.

According to previous works, autophagy inhibits EMT and metastasis in gastric cancer [32,39]. Autophagy impairs the migration and invasion of cancer cells by downregulating Snail and Slug [40]. Conversely, silencing autophagy-related proteins restores the mesenchymal phenotype [32,33,39]. Similarly, our study shows that starvation-induced autophagy leads to the degradation of intracellular SNAI1 and the suppression of cancer progression. In contrast, blocking autophagy with chemicals or by gene silencing increases SNAI1 protein levels and leads to normal cancer progression. Moreover, other studies have suggested that autophagy attenuates EMT and tumor metastasis by the degradation of Twist1 and SNAI1 $[33,41]$. In addition to cancers, autophagy-dependent SNAI1 degradation also 
determines hepatocyte identity by regulating the transition between EMT and MET. Indeed, stimulation of autophagy in hepatocytes strongly affects EMT by promoting SNAI1 degradation, and conversely, TGF $\beta$-induced EMT leads to autophagy impairment [42]. These all studies suggest that autophagy might play a key role in controlling the intracellular level of SNAI1, which is essential to determine cell transitions in cancer and hepatocyte differentiation.

SNAI1 acts as a main driver of EMT and metastasis [17-19]. It specifically inhibits the transcription of anticancer genes such as CDH1/E-cadherin and activates mesenchymal markers to promote the metastasis of most cancers [20-23]. Our results show that the intracellular levels of E-cadherin decrease when cancer cells are starved and are increased by the inhibition of autophagy, suggesting that autophagy-dependent SNAI1 degradation is directly associated with its downstream targets such as E-cadherin. Additionally, other EMT-related proteins including N-cadherin, and Zeb1 are likely regulated in an autophagy-dependent manner (Figure 3).

In this study, we suggest that SNAI1 directly binds to LC3 in cancer cells. LC3 plays a key role in the initiation of autophagosome formation. It also mediates autophagy by its association with specific proteins (e.g., SQSTM1) through a highly conserved protein domain, called an LIR. The LIR contains two hydrophobic amino acids (e.g., tryptophan, W; leucine, L; and phenylalanine, Y) separated by two nonspecific amino acids (X). Some proteins that possess an LIR are selectively degraded by autophagy through a direct link with LC3, whereas other ubiquitinated proteins are engulfed by an autophagosome via an indirect association with the LC3-SQSTM1 complex. Interestingly, we observed that the amino acid sequence of SNAI1 contains three putative LIR motifs $\left({ }^{61} \mathrm{WXXV}{ }^{64},{ }^{74} \mathrm{WXXL} \mathrm{L}^{77}\right.$, and ${ }^{163} \mathrm{YXXL}^{166}$ ), and that SNAI1 was physically associated with LC3 in a coimmunoprecipitation assay and by a colocalization analysis (Figure 2). In addition, SNAI1 was associated with SQSTM1, indicating that SNAI1 can be removed by autophagy in an SQSTM1-dependent manner. Based on these results, we suggest that intracellular SNAI1 protein levels are controlled by autophagy during cancer progression, which is consistent with results from previous studies in which autophagy inhibited cancer growth by degrading SNAI1 [32,33].

Both EMT and cancer metastasis are stimulated by an increase in SNAI1, N-cadherin, Zeb1, and vimentin, and a decrease in E-cadherin [17-23]. In this study, we show a similar decrease in SNAI1, N-cadherin, and Zeb1 upon starvation-induced autophagy in two cancer cell lines. Conversely, E-cadherin levels were increased by the induction of autophagy in cancer cells (Figure 3). Since three other EMT-related proteins (N-cadherin, Zeb1, and vimentin) are downstream mediators of SNAI1, they should be dependent on the induction of autophagy. Indeed, the intracellular levels of these three proteins were also regulated by autophagy, in contrast to that of SNAI1. These three proteins were inhibited by the induction of autophagy and increased in response to the inhibition of autophagy. These results agree with those of Catalano et al. [32] and other studies [42,43] in which autophagy was found to reverse EMT. The connections between EMT-related proteins and their association with autophagy are presented in Figure 7.

In conclusion, SNAI1 is selectively degraded during starvation-induced autophagy by its interaction with SQSTM1 or LC3. SNAI1 degradation inhibits cancer progression by downregulating many EMT-related proteins. As a result, autophagy-induced SNAI1 degradation in the cytoplasm inhibits SNAI1 translocation into the nucleus and blocks the transcription of many genes involved in cancer cell invasion and migration. This study suggests that autophagy could be a possible target for the development of therapeutic strategies to inhibit cancer progression and modulate the stem cell-like properties and chemoresistance of cancer cells. 


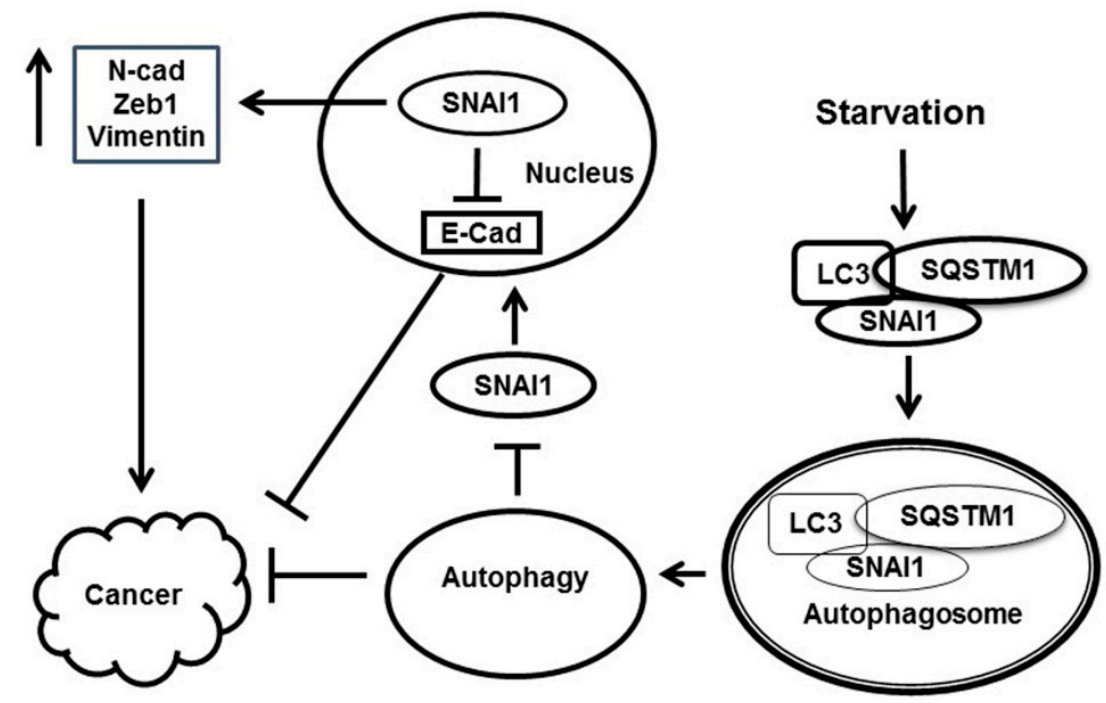

Figure 7. Schematic representation of autophagy-dependent SNAI1 degradation during cancer progression. Starvation-induced autophagy leads to the degradation of SNAI1 via the incorporation of LC3-SQSTM1-SNAI1 complexes into autophagosomes. Consequently, the low levels of nuclear SNAI1 cause a decrease in the levels of EMT-related proteins (N-cadherin, Zeb1, and vimentin) and an increase in E-cadherin through transcriptional deactivation. Thus, the induction of autophagy inhibits cancer progression.

Author Contributions: Conceptualization, S.Z., and D.K.; methodology, S.Z., J.H., and D.K.; data investigation and analysis, S.Z., J.H., T.H.L., M.A., T.M.P.; writing preparation and editing, S.Z., J.H., T.H.L., M.A., T.M.P., and D.K.; supervision, D.K.

Funding: This study was supported by Development Fund Foundation, Gyeongsang National University, 2015 and the Basic Research Program of the National Research Foundation of Korea (NRF) and funded by the Ministry of Education Science and Technology (2018R1D1A1B07043715) and the Ministry of Science, ICT and Future Planning (NRF-2015R1A5A2008833).

Conflicts of Interest: The authors declare no conflicts of interest.

\section{Abbreviations}

ATG, autophagy-related gene; DAPI, 4',6-diamidine-2'-phenylindole dihydrochloride; DMEM, Dulbecco's modified Eagle's medium; EMT, epithelial-to-mesenchymal transition; FBS, fetal bovine serum; FITC, fluorescein isothiocyanate; HBSS, Hank's buffered salt solution; LIR, LC3-interacting region; MAP1LC3/LC3, microtubule-associated proteins 1 light chain 3; PBS, phosphate-buffered saline; PI, propidium iodide; RPMI, Roswell Park Memorial Institute 1640 medium; ULK1, Unc-51 like autophagy-activating kinase 1; SQSTM1/p62, sequestosome-1; SDS-PAGE, sodium dodecyl sulfate-polyacrylamide gel electrophoresis

\section{References}

1. Mizushima, N.; Levine, B.; Cuervo, A.M.; Klionsky, D.J. Autophagy fights disease through cellular self-digestion. Nature 2008, 451, 1069-1075. [CrossRef]

2. Green, D.R.; Galluzzi, L.; Kroemer, G. Mitochondria and the autophagy-inflammation-cell death axis in organismal aging. Science 2011, 333, 1109-1112. [CrossRef]

3. Levine, B.; Klionsky, D.J. Development by self-digestion: molecular mechanisms and biological functions of autophagy. Dev. Cell 2004, 6, 463-477. [CrossRef]

4. Klionsky, D.J. Autophagy: from phenomenology to molecular understanding in less than a decade. Nat. Rev. Mol. Cell Biol. 2007, 8, 931-937. [CrossRef] [PubMed]

5. White, E.; DiPaola, R.S. The double-edged sword of autophagy modulation in cancer. Clin. Cancer Res. 2009, 1078-5023. [CrossRef] [PubMed]

6. White, E. Deconvoluting the context-dependent role for autophagy in cancer. Nat. Rev. Cancer 2012, 12, 401-410. [CrossRef] [PubMed] 
7. Kroemer, G.; Mariño, G.; Levine, B. Autophagy and the integrated stress response. Mol. Cell 2010, 40, $280-293$. [CrossRef] [PubMed]

8. Mehlen, P.; Puisieux, A. Metastasis: a question of life or death. Nat. Rev. Cancer 2006, 6, 449-458. [CrossRef] [PubMed]

9. Valastyan, S.; Weinberg, R.A. Tumor metastasis: molecular insights and evolving paradigms. Cell 2011, 147, 275-292. [CrossRef] [PubMed]

10. Lambert, A.W.; Pattabiraman, D.R.; Weinberg, R.A. Emerging biological principles of metastasis. Cell 2017, 168, 670-691. [CrossRef] [PubMed]

11. Gupta, G.P.; Massagué, J. Cancer metastasis: building a framework. Cell 2006, 127, 679-695. [CrossRef] [PubMed]

12. Eccles, S.A.; Welch, D.R. Metastasis: recent discoveries and novel treatment strategies. Lancet 2007, 369, 1742-1757. [CrossRef]

13. Fidler, I.J. The pathogenesis of cancer metastasis: the'seed and soil'hypothesis revisited. Nat. Rev. Cancer 2003, 3, 453-458. [CrossRef] [PubMed]

14. Nieto, M.A.; Huang, R.Y.-J.; Jackson, R.A.; Thiery, J.P. EMT: 2016. Cell 2016, 166, 21-45. [CrossRef] [PubMed]

15. Lamouille, S.; Xu, J.; Derynck, R. Molecular mechanisms of epithelial-mesenchymal transition. Nat. Rev. Mol. Cell Biol. 2014, 15, 178-196. [CrossRef] [PubMed]

16. Zheng, X.; Carstens, J.L.; Kim, J.; Scheible, M.; Kaye, J.; Sugimoto, H.; Wu, C.-C.; LeBleu, V.S.; Kalluri, R. Epithelial-to-mesenchymal transition is dispensable for metastasis but induces chemoresistance in pancreatic cancer. Nature 2015, 527, 525-530. [CrossRef] [PubMed]

17. Dave, N.; Guaita-Esteruelas, S.; Gutarra, S.; Frias, À.; Beltran, M.; Peiro, S.; de Herreros, A.G. Functional cooperation between Snail1 and Twist in the regulation of Zeb1 expression during epithelial-to-mesenchymal transition. J. Biol. Chem. 2011, 286, 12024-12032. [CrossRef]

18. Tran, D.D.; Corsa, C.A.S.; Biswas, H.; Aft, R.; Longmore, G.D. Temporal and spatial cooperation of Snail1 and Twist1 during epithelial-mesenchymal transition predicts for human breast cancer recurrence. Mol. Cancer Res. 2011, 9, 1644-1657. [CrossRef]

19. Peinado, H.; Olmeda, D.; Cano, A. Snail, Zeb and bHLH factors in tumour progression: an alliance against the epithelial phenotype? Nat. Rev. Cancer 2007, 7, 415-428. [CrossRef]

20. Batlle, E.; Sancho, E.; Francí, C.; Domínguez, D.; Monfar, M.; Baulida, J.; de Herreros, A.G. The transcription factor snail is a repressor of E-cadherin gene expression in epithelial tumour cells. Nat. Cell Biol. 2000, 2, 84-89. [CrossRef]

21. Cano, A.; Pérez-Moreno, M.A.; Rodrigo, I.; Locascio, A.; Blanco, M.J.; del Barrio, M.G.; Portillo, F.; Nieto, M.A. The transcription factor snail controls epithelial-mesenchymal transitions by repressing E-cadherin expression. Nat. Cell Biol. 2000, 2, 76-83. [CrossRef] [PubMed]

22. Wang, X.; Zheng, M.; Liu, G.; Xia, W.; McKeown-Longo, P.J.; Hung, M.-C.; Zhao, J. Krüppel-like factor 8 induces epithelial to mesenchymal transition and epithelial cell invasion. Cancer Res. 2007, 67, 7184-7193. [CrossRef] [PubMed]

23. Nieto, M.A. The snail superfamily of zinc-finger transcription factors. Nat. Rev. Mol. Cell Biol. 2002, 3, 155-166. [CrossRef] [PubMed]

24. Kurrey, N.K.; Jalgaonkar, S.P.; Joglekar, A.V.; Ghanate, A.D.; Chaskar, P.D.; Doiphode, R.Y.; Bapat, S.A. Snail and slug mediate radioresistance and chemoresistance by antagonizing p53-mediated apoptosis and acquiring a stem-like phenotype in ovarian cancer cells. Stem Cells 2009, 27, 2059-2068. [CrossRef] [PubMed]

25. Eyler, C.E.; Rich, J.N. Survival of the fittest: cancer stem cells in therapeutic resistance and angiogenesis. J. Clin. Oncol. Off. J. Am. Soc. Clin. Oncol. 2008, 26, 2839-2845. [CrossRef] [PubMed]

26. Mani, S.A.; Guo, W.; Liao, M.-J.; Eaton, E.N.; Ayyanan, A.; Zhou, A.Y.; Brooks, M.; Reinhard, F.; Zhang, C.C.; Shipitsin, M. The epithelial-mesenchymal transition generates cells with properties of stem cells. Cell 2008, 133, 704-715. [CrossRef] [PubMed]

27. Lee, S.-H.; Lee, S.-J.; Chung, J.Y.; Jung, Y.S.; Choi, S.Y.; Hwang, S.H.; Choi, D.; Ha, N.C.; Park, B.J. p53, secreted by K-Ras-Snail pathway, is endocytosed by K-Ras-mutated cells; implication of target-specific drug delivery and early diagnostic marker. Oncogene 2009, 28, 2005-2014. [CrossRef]

28. Tung, Y.-T.; Hsu, W.-M.; Lee, H.; Huang, W.-P.; Liao, Y.-F. The evolutionarily conserved interaction between LC3 and p62 selectively mediates autophagy-dependent degradation of mutant huntingtin. Cell. Mol. Neurobiol. 2010, 30, 795-806. [CrossRef] 
29. Bjørkøy, G.; Lamark, T.; Brech, A.; Outzen, H.; Perander, M.; Øvervatn, A.; Stenmark, H.; Johansen, T. p62/SQSTM1 forms protein aggregates degraded by autophagy and has a protective effect on huntingtin-induced cell death. J. Cell Biol. 2005, 171, 603-614. [CrossRef]

30. Pankiv, S.; Clausen, T.H.; Lamark, T.; Brech, A.; Bruun, J.-A.; Outzen, H.; Øvervatn, A.; Bjørkøy, G.; Johansen, T. p62/SQSTM1 binds directly to Atg8/LC3 to facilitate degradation of ubiquitinated protein aggregates by autophagy. J. Biol. Chem. 2007, 282, 24131-24145. [CrossRef]

31. Shvets, E.; Fass, E.; Scherz-Shouval, R.; Elazar, Z. The N-terminus and Phe52 residue of LC3 recruit p62/SQSTM1 into autophagosomes. J. Cell Sci. 2008, 121, 2685-2695. [CrossRef] [PubMed]

32. Catalano, M.; D’alessandro, G.; Lepore, F.; Corazzari, M.; Caldarola, S.; Valacca, C.; Faienza, F.; Esposito, V.; Limatola, C.; Cecconi, F. Autophagy induction impairs migration and invasion by reversing EMT in glioblastoma cells. Mol. Oncol. 2015, 9, 1612-1625. [CrossRef] [PubMed]

33. Lv, Q.; Hua, F.; Hu, Z.-W. DEDD, a novel tumor repressor, reverses epithelial-mesenchymal transition by activating selective autophagy. Autophagy 2012, 8, 1675-1676. [CrossRef]

34. Vinas-Castells, R.; Frias, A.; Robles-Lanuza, E.; Zhang, K.; Longmore, G.D.; García de Herreros, A.; Díaz, V.M. Nuclear ubiquitination by FBXL5 modulates Snail1 DNA binding and stability. Nucleic Acids Res. 2013, 42, 1079-1094. [CrossRef] [PubMed]

35. Jin, Y.; Shenoy, A.K.; Doernberg, S.; Chen, H.; Luo, H.; Shen, H.; Lin, T.; Tarrash, M.; Cai, Q.; Hu, X. FBXO11 promotes ubiquitination of the Snail family of transcription factors in cancer progression and epidermal development. Cancer Lett. 2015, 362, 70-82. [CrossRef] [PubMed]

36. Viñas-Castells, R.; Beltran, M.; Valls, G.; Gómez, I.; García, J. M.; Montserrat-Sentís, B.; Baulida, J.; Bonilla, F.; de Herreros, A.G.; Díaz, V.M. The hypoxia-controlled FBXL14 ubiquitin ligase targets SNAIL1 for proteasome degradation. J. Biol. Chem. 2010, 285, 3794-3805. [CrossRef] [PubMed]

37. Noda, N.N.; Ohsumi, Y.; Inagaki, F. Atg8-family interacting motif crucial for selective autophagy. FEBS Lett. 2010, 584, 1379-1385. [CrossRef]

38. Ichimura, Y.; Kirisako, T.; Takao, T.; Satomi, Y.; Shimonishi, Y.; Ishihara, N.; Mizushima, N.; Tanida, I.; Kominami, E.; Ohsumi, M. A ubiquitin-like system mediates protein lipidation. Nature 2000, 408, 488-492. [CrossRef]

39. Qin, W.; Li, C.; Zheng, W.; Guo, Q.; Zhang, Y.; Kang, M.; Zhang, B.; Yang, B.; Li, B.; Yang, H. Inhibition of autophagy promotes metastasis and glycolysis by inducing ROS in gastric cancer cells. Oncotarget 2015, 6, 39839-39854. [CrossRef]

40. Gugnoni, M.; Sancisi, V.; Manzotti, G.; Gandolfi, G.; Ciarrocchi, A. Autophagy and epithelial-mesenchymal transition: an intricate interplay in cancer. Cell Death Dis. 2016, 7, e2520. [CrossRef]

41. Qiang, L.; Zhao, B.; Ming, M.; Wang, N.; He, T.C.; Hwang, S.; Thorburn, A.; He, Y.Y. Regulation of cell proliferation and migration by p62 through stabilization of Twist1. Proc. Natl. Acad. Sci. U S A. 2014, 111, 9241-9246. [CrossRef] [PubMed]

42. Grassi, G.; Di Caprio, G.; Santangelo, L.; Fimia, G.M.; Cozzolino, A.M.; Komatsu, M.; Ippolito, G.; Tripodi, M.; Alonzi, T. Autophagy regulates hepatocyte identity and epithelial-to-mesenchymal and mesenchymal-to-epithelial transitions promoting Snail degradation. Cell Death Dis. 2015, 6, e1880. [CrossRef] [PubMed]

43. Gugnoni, M.; Sancisi, V.; Gandolfi, G.; Manzotti., G.; Ragazzi, M.; Giordano, D.; Tamagnini, I.; Tigano, M.; Frasoldati, A.; Piana, S.; Ciarrocchi, A. Cadherin-6 promotes EMT and cancer metastasis by restraining autophagy. Oncogene 2017, 36, 667-677. [CrossRef] [PubMed]

(C) 2019 by the authors. Licensee MDPI, Basel, Switzerland. This article is an open access article distributed under the terms and conditions of the Creative Commons Attribution (CC BY) license (http://creativecommons.org/licenses/by/4.0/). 